\title{
DEUX REPRÉSENTATIONS DE « L'AUTRE » EN FRANCE SOUS L'ANCIEN RÉGIMe : LA PÉRUVIENNE de Graffigny (1747) ET LE HURON DE VOLTAIRE (1767).
}

\author{
Jitka RADIMSKÁ \\ Université de Bohême du Sud, České Budějovice
}

\begin{abstract}
En): The article brings a complementary view of two " bestsellers" of the $18^{\text {th }}$ century, published anonymously but soon reedited under the names of their authors: the epistolary novel Lettres d'une Péruvienne by Mme de Graffigny (1947) and "a real story" L'Ingénu by Voltaire (1767). Both texts, severely criticised by some but enthuasiasticaly accepted by others, deserve being resituated in their century as they mirror back its reflection. This is the perspective through which observe the editorial, generic and narative stategies used by Mme de Graffigny and Voltaire in their outstandingly successful editions.
\end{abstract}

Résumé (Fr) : L'étude apporte un regard complémentaire sur deux «best-sellers » du XVIII ${ }^{\mathrm{e}}$ siècle, publiés sous l'anonymat, mais bientôt réédités sous le nom des auteurs: le roman épistolaire Lettres d'une Péruvienne de Mme de Graffigny (1947) et «l'histoire véritable» L'Ingénu de Voltaire (1767). Critiqués sévèrement par les uns, accueillis avec enthousiasme par d'autres, les deux textes méritent d'être resitués dans leur siècle dont ils renvoient le reflet. C'est dans cette perspective que nous nous proposons d'observer les stratégies éditoriale, générique et narrative, adoptées par Mme de Graffigny et Voltaire dans leurs remarquables succès d'édition.

Keywords (En): Lettres d'une Péruvienne (1747); Mme de Graffigny (1695-1758) ; L'Ingénu (1767) ; Voltaire (1694-1778) ; noble savage; editorial strategy; stranger sociability; the mirror effect

Mots-clés (Fr) : Lettres d'une Péruvienne (1747) ; Mme de Graffigny (1695-1758) ; L'Ingénu (1767); Voltaire (1694-1778); le bon sauvage; la stratégie éditoriale; la sociabilité de l'étranger ; l'effet de miroir

Pendant la deuxième moitié du XVIII ${ }^{\mathrm{e}}$ siècle, quand la légitimité du roman commença à être contestée par la critique, les romanciers tentèrent de s'exprimer explicitement sur ce qu'ils proposaient à lire à un lectorat déjà assez différencié. Parmi eux, Mme de Graffigny, femme de lettres débutant à l'âge mûr, et Voltaire, écrivain censuré, mais reconnu. Les deux auteurs finirent par trouver une forme convenable pour leurs fictions romanesques plus ou moins vraisemblables, dans lesquelles ils relatèrent l'histoire d'un(e) 'bon(ne) sauvage' venu(e) dans l'Ancienne France du Nouveau Monde. Dès la parution des ouvrages, ils préfèrèrent laisser les lecteurs dans l'incertitude sur l'identité de l'auteur des manuscrits livrés aux libraires. Ils s'efforcèrent de souligner l'authenticité de leurs récits et invitèrent les lecteurs français à suivre les péripéties de protagonistes étrangers, pour qu'ils pussent savourer avec eux le goût des temps perdus. Ainsi, ils réussirent à faire entrer le public des Lumières dans les débats sur la vie littéraire complexe de leur époque dont ils étaient, chacun à sa manière, acteurs actifs. 


\section{Du côté des femmes dépourvues de tout...}

Mme de Graffigny semblait s'être assuré une grande renommée dans la littérature française dès la parution de son roman épistolaire, en 1747, parce qu'elle savait joindre à l'attrait sentimental des Lettres portugaises (1669) une analyse de la société des Lettres persanes (1721). Cependant, dès la dernière année de sa vie sa célébrité diminuait et, vers 1800, l'écrivaine se vit dépouillée de tout son prestige. Cinquante ans plus tard, dans l'une de ses «Causeries du lundi», Sainte-Beuve parlait moins de son œuvre littéraire, passée de mode, que de ses lettres de Cirey où elle avait été reçue par la marquise du Châtelet et Voltaire. Sainte-Beuve finit par constater que «sa vie fut un roman plus touchant que ceux qu'elle a écrits ». (Cité d'après LAFFONT, BOMPIANI, 1986 : 368-369)

Voilà pourquoi nous préférons remonter vers le début du $\mathrm{XIX}^{\mathrm{e}}$ siècle et citer, ci-dessous, un extrait biographique sur Mme de Graffigny, qui semble mieux caractériser les enjeux et les défis de son roman par lettres dont la parution suscita tant de réactions contraires. On peut y apprécier l'équilibre des pour et des contre dans la présentation de l'ouvrage et le ton neutre et non partial. Dans la partie des atouts, le biographe met en relief l'originalité de la démarche de l'épistolière concernant la structure et le style, l'effet miroir qui organise le tout et illustre bien l'idée principale de l'œuvre. La partie des défauts reprend les opinions et les formules des comptes rendus contemporains : dénouement inopiné, anachronismes intolérables, propos métaphysiques ou exaltation exagérée inadmissibles pour une jeune femme non civilisée. Pourtant, la déception des doctes n'empêcha point les lecteurs d'éprouver de la joie lors de la lecture, ni à l'écrivaine, de la satisfaction d'avoir réussi à accomplir leur horizon d'attente.

"Mme de Graffigny débuta en littérature par une nouvelle imprimée dans le Recueil de ces Messieurs. Ce morceau, intitulé Nouvelle espagnole: Le mauvais exemple produit autant de vertus que de vices (1745), avait une fausse maxime pour titre, et un abus de maximes gâtait le cours du récit, quoique mêlé de morceaux où l'on trouvait du sentiment. Elle répondit aux critiques par les Lettres Péruviennes, qui ont fait sa réputation. L'idée et le cadre de cet ouvrage sont également ingénieux : des sentiments vrais, naïfs, autant que passionnés, des descriptions charmantes, une adresse peu connue à embellir les moindres objets, et à tirer parti de la situation bizarre de la jeune Péruvienne Zilia, transportée tout à coup au milieu d'un monde dont les mours et les usages lui sont totalement étrangers (adresse qui rajeunit aux yeux du lecteur les objets les plus familiers, par la peinture qu'en fait Zilia, et les impressions qu'elle en reçoit) ; voilà les avantages qui firent le succès des Lettres péruviennes dans le temps, et leur ont assuré pour toujours. Voici également les critiques qu'essuya Mme de Graffigny: le dénouement ne satisfait pas; l'illusion est parfois détruite par les anachronismes de l'auteur sur des usages qui appartiennent à son temps et qui étaient ignorés dans celui où elle place le voyage de la Péruvienne en France. Son style élégant et naturel est trop souvent déparé par les traits métaphysiques qu'elle y prodigue; et ce défaut, déjà assez considérable dans un ouvrage de sentiment, n'est ni pardonnable à Zilia, que l'auteur fait parler, ni concevable chez un auteur de 55 ans : c'est proprement celui du jeune âge. L'expérience et un goût épuré ramènent à la nature. La jeunesse, pleine d'exaltation, a dû chercher au-delà de l'expression du vrai du langage qui puisse satisfaire cette exaltation: l'âge mûr ne connaît de beau que le vrai; le style comme l'esprit y sentent l'homme désabusé. Les taches dont nous venons de parler, et quelques méprises de noms et de termes péruviens, pardonnables à une femme, n'empêchèrent pas le public de rendre justice à l'auteur des Lettres péruviennes. » (Biographie universelle ancienne et moderne, $1817: 262-264)$ 
Les Lettres péruviennes parurent pour la première fois en 1747 avec une fausse adresse et sous l'anonymat. En 1752, une seconde édition augmentée de trois lettres et d'une introduction historique parut avec le privilège du Roi sous le nom de l'écrivaine. Le succès fut immense : 50 éditions, rééditions ou contrefaçons entre 1747 et 1800 et, durant la même période, 40 éditions ou rééditions de traductions, parmi lesquelles les traductions princeps en anglais (1748), italien (1754), allemand (1760), polonais (1784), russe (1791) et espagnol (1792), poursuivies plus tard par celles en portugais (1802), suédois (1844) et danois (1855). Plusieurs de ces textes incorporèrent trois suites diverses, parues en 1748 ou à la fin du siècle. Mis à l'Index en 1765, le roman fut ignoré, méconnu et méprisé pendant plusieurs décennies. Ce n'est que dans les années quatre-vingts du $\mathrm{XX}^{\mathrm{e}}$ siècle que la modernité des Lettres d'une Péruvienne a été reconnue. Elle consiste à laisser la femme, d'abord sujette, inventer et construire elle-même son propre destin :

"L'important est de suggérer à la femme déracinée, délaissée (Aza, finalement retrouvé, n'aime plus sa Péruvienne), persécutée (en l'occurrence par les propositions du Français Déterville) une forme de bonheur par défaut, qui préserve son équilibre au sein d'une société hostile ». (BeAumarchais, Couty, Rey, 1994 : 1042)

De nos jours, les lecteurs francophones ont à la disposition deux éditions critiques et annotées du roman épistolaire de Mme de Graffigny : celle de Jonathan Mallinson d'Oxford (2002), et celle de Rotraud von Koulessa d'Augsbourg (2014). L'édition critique de la correspondance privée de l'écrivaine en 15 volumes a été préparée par une équipe de spécialistes du monde entier (1985-2016). Ainsi, nous pouvons lire Mme de Graffigny pour elle-même et comprendre les affaires de l'époque. Rappelons que l'initiative visant à découvrir la particularité de l'écriture de Mme de Graffigny, femme écrivaine vivant de sa plume, et l'intérêt manifesté pour son œuvre venaient des pays étrangers.

Mallinson situe le roman par lettres de Mme de Graffigny dans le champs littéraire de l'époque des Lumières. Il récapitule les débats reflétant la réception contemporaine de l'ouvrage et compare l'édition princeps avec celles des années 1750 et 1752, augmentées d'une introduction à l'histoire du Pérou et de quelques lettres clés par Mme de Graffigny. Mallinson montre les innovations de la démarche de l'écrivaine en faisant la comparaison avec le roman traditionnel de l'âge baroque et classique. Il examine la jeune Péruvienne sous toutes ses faces: en tant qu'amante, étrangère, philosophe, femme et épistolière pour conclure que l'une des caractéristiques les plus frappantes du roman est la fonction multiple de l'héroïne et l'originalité de son discours, tant sur le plan thématique que formel. En annexe, Mallinson fait revue des suites, traductions et continuations postérieures de l'œuvre en question et ajoute un dossier de textes relatifs à la réception des Lettres Péruviennes au XVIII ${ }^{\mathrm{e}}$ siècle.

Rotraud von Koulessa a soutenu sa thèse sur Mme de Graffigny en 1997. Dans la version française, parue chez Garnier en 2016, elle situe le roman dans le contexte des pièces dramatiques de Mme de Graffigny et augmente son édition critique par des suites librement adaptées au XVIII ${ }^{\mathrm{e}}$ siècle : trois textes dramatiques de Mme de 
Graffigny (Cénie, Ziman et Zenise suivi de Phaza, 1750, 1770), deux adaptations scéniques (La Péruvienne de Chabannes, 1754, La Peruviana, de Goldoni, 1755) et une suite de Montbart (Lettres Taïtiennes, 1786). Elle se concentre sur l'analyse des stratégies littéraires du roman épistolaire, l'exotisme et la sensibilité féminine, ainsi que sur la genèse du livre. Elle s'appuie sur la correspondance privée de l'écrivaine avec son admirateur lorrain François-Antoine Devaux, poète galant et improvisateur brillant, connu sous le nom de Panpan. Rotraud von Koulessa rappelle brièvement la réception de l'œuvre après 1800 et le mépris manifesté par la critique française au XIX ${ }^{\mathrm{e}}$ siècle. Elle finit par une synthèse de ces débats nés sous la plume de Jean Larnac, en 1929, qui attribuait à l'écrivaine le mérite d'avoir inauguré la mode du roman épistolaire avant Rousseau.

Revenons à la question de la stratégie éditoriale de l'édition remaniée des Lettres en 1752, dans laquelle Mme de Graffigny trouvait indispensable d'avertir les lecteurs de toutes ses démarches. En réaction à la critique contemporaine, elle réécrivit certains passages du point de vue stylistique, corrigea des erreurs de fait, précisa certaines idées abstraites. Elle ajouta des notes explicatives, quelques références de page, et surtout l'Introduction historique qui donnait au roman un aspect plus érudit. Elle y décrivait la culture particulière des Incas pour mieux expliquer le comportement de son héroïne. Elle ne cachait point l'intention formative de son ouvrage :

«Nous méprisons les Indiens; à peine accordons-nous une âme pensante à ces peuples malheureux; cependant leur histoire est entre les mains de tout le monde; nous y trouvons partout des monuments de la sagacité de leur esprit, et de la solidité de leur philosophie.» (GRAFFIGNY, 2002 : 99)

Par contre, elle renonça fermement à suivre les propositions de dénouements alternatifs: pathétique, mort de Zilia (recommandé par Pierre Clément), ou conventionnel du roman sentimental, mariage avec Aza et amitié de Déterville (argumenté par Anne-Robert-Jacques Turgot). Certains changements dans la présentation du texte l'accommodèrent au goût de l'époque, par exemple les illustrations des scènes clés, choisies par l'écrivaine elle-même, qui rapprochaient deux cultures diverses (lettre XII où Zilia adopte pour la première fois un habit français). Ces changements montrent son désir d'influencer la façon dont on lisait les fictions romanesques. De plus, l'écrivaine réaffirma ainsi son indépendance littérale et métaphorique.

En ce qui concerne Zilia, elle diffère nettement des protagonistes venus d'ailleurs, surtout par son destin malheureux parce qu'elle doit affronter des difficultés que les autres ne rencontrent pas : sans racines, sans argent, sans les moyens de se définir. Elle se rend compte de son statut incertain et de sujette dans la société française : "Je n'ai ni or, ni terres, ni industrie, je fais nécessairement partie des citoyens de cette ville. Ô ciel! dans quelle classe dois-je me ranger?» (Lettre XX) Au fil du récit, elle garde sa propre identité, elle exprime, explique et évalue ses découvertes par rapport à sa propre culture. C'est l'écriture qui devient la condition sine qua non de son existence nouvelle. Pour décrire les objets inexistants dans son pays, elle recourt à des tournures périphrastiques, comme par 
ex. «maison flottante ». Significative de ce point de vue est la lettre où, étant entrée dans la chambre de son nouveau domicile, elle crut rencontrer son homologue, mais il s'agit de son portrait qu'elle vit se mirer dans la glace :

«j'ai vu dans l'enfoncement une jeune personne habillée comme une Vierge du Soleil ; j'ai couru à elle les bras ouverts. Quelle surprise, mon cher Aza, quelle surprise extrême, de ne trouver qu'une résistance impénétrable, où je voyais une figure humaine se mouvoir dans un espace fort étendu. »

Et elle prolonge sa réflexion ainsi :

«Ces prodiges troublent la raison, ils offusquent le jugement; que faut-il penser des habitants de ce pays ; Faut-il les craindre, faut-il les aimer ; Je me garderai bien de rien déterminer làdessus. » (Lettre $\mathrm{X})$

L'effet miroir cependant constitue l'élément de base qui opère toute description de l'épistolière, tant sur le plan thématique que formel. Les lettres de Zilia ne sont pas détachables du texte.

Avec la vingtième lettre, Zilia ouvre un nouveau thème extrêmement important pour sa formation intellectuelle, la lecture : «Jusqu'ici, mon cher Aza, tout occupée des peines de mon cour, je ne t'ai point parlé de celles de mon esprit. » (Lettre XX) Au fur et à mesure, après avoir appris le français, elle se livre à la lecture méthodique et à l'étude. Elle découvre ainsi la source du savoir et le goût de s'instruire :

"Je dois une partie de ces connaissances à une sorte d'écriture que l'on appelle Livres; quoique je trouve encore beaucoup de difficultés à comprendre ce qu'ils contiennent, ils me sont fort utiles, j'en tire des notions, Céline m'explique ce qu'elle en sait, et j'en compose des idées que je crois justes. »

Dans les premières lettres, Zilia raconte sa séparation d'Aza au moyen de métaphores transparentes. Quand elle exprime son désir de comprendre le pays qui l'entoure, elle se sert du terme «lumière » (Lettre IX). Elle se sert de la même métaphore pour exprimer la satisfaction intellectuelle que lui procuraient les livres français :

"Quelques-uns de ces Livres apprennent ce que les hommes ont fait, et d'autres ce qu'ils ont pensé. Je ne puis t'exprimer, mon cher Aza, l'excellence du plaisir que je trouverais à les lire, si je les entendais mieux, ni le désir extrême que j'ai de connaître quelques-uns des hommes divins qui les composent. Je comprends qu'il sont à l'âme ce que le Soleil est à la terre, et que je trouverais avec eux toutes les lumières, tous les secours dont j'ai besoin, mais je ne vois nul espoir d'avoir jamais cette satisfaction. »

Nous tenons à souligner son admiration pour des auteurs de livres qui méritent, d'après elle, plus de récompense et de notoriété de leurs efforts : «Quoique Céline lise assez souvent, elle n'est pas assez instruite pour me satisfaire ; à peine avaitelle pensé que les Livres fussent faits par des hommes, elle en ignore les noms, et même s'ils vivent encore. » Ainsi, Zilia reconnaît aux Français une valeur intellectuelle et leur génie inventif. Les gens des Lumières purent satisfaire un désir de connaître qui n'a pas de bornes. 


\section{Du côté des hommes sans nom...}

Comme Mme de Graffigny, Voltaire découvrit sa prédilection pour le genre narratif tardivement, à l'âge mûr. Ses contes cachaient généralement, sous des fictions d'ordre naturel ou merveilleux, quelques principes de philosophie spéculative, ou quelque vérité de morale pratique. Dans la Biographie universelle des années vingt du XIX ${ }^{\mathrm{e}}$ siècle, l'article sur Voltaire comprend 46 pages. Le biographe situe ses récits narratifs dans le contexte de son œuvre, tout en hésitant à les qualifier de romans. Il apprécie l'art de dépeindre de Voltaire, qui relie avec habilité des concepts opposés : la raison et l'esprit, le plaisant et le pathétique, la réalité et sa perception. Vu l'autorité littéraire de Voltaire, qui influençait largement l'opinion publique, il constate à regret, que son naturel critique et son inclination à railler ses adversaires imprègnent souvent ses compositions de trois espèces de travers critiquables : allusions irréligieuses, animosité personnelle et plaisanteries d'un cynisme de mauvais goût.

Il y a peu de lectures aussi attrayantes que celles des romans de Voltaire. Moins étendues que les compositions qu'on nomme ainsi ordinairement, on les appellerait volontiers des contes : plusieurs pour le genre ressemblent à ceux que l'Orient nous a transmis, et quelques-uns sont, en partie, des emprunts faits à la littérature anglaise. Presque tous ont un but philosophique. L'Ingénu n'est pas de cette unité-là. C'est un tissu d'aventures dont chacune, ainsi que tout événement de la vie, porte avec soi son instruction. La raison et l'esprit, le plaisant et le pathétique, y sont mêlés et fondus avec cet art facile et heureux qui constitue proprement la manière de Voltaire. Pour faire entrer dans un même cadre les mours contrastées de plusieurs peuples divers, genre de peinture où il excellait, Voltaire fait voyager au loin les héros de presque tous ses romans. Les objets, vus par un étranger, tels qu'ils sont dans la réalité, et non tels que l'accoutumance les fait paraître aux yeux des habitants du pays, sont présentés naturellement sous leur aspect le plus philosophique et le plus piquant. On rencontre à regret, dans ces compositions charmantes, quelques allusions irréligieuses, quelques traits d'animosité personnelle, et quelques plaisanteries d'un cynisme de mauvais goût, trois espèces de taches dont on peut dire que n'est exempt presque aucun des ouvrages de la vieillesse de l'auteur. Voltaire ne voulut ou plutôt ne put rester indifférent à rien de ce qui est du ressort de la pensée. Mais ses passions obscurcirent et faussèrent trop souvent sa raison. Il voulait détruire les anciens préjugés des peuples, et il mettait les siens à la place. Sa haine contre les prêtres ne se cache pas toujours assez adroitement sous les apparences d'un ardent amour pour l'humanité et, dans sa passion contre la Genèse, il attaque quelquefois la saine physique, en croyant n'attaquer que les livres sacrés. (Biographie universelle ancienne et moderne, 1827 : 501-502)

L'autorisation dite «tacite » de publier Le Huron, ou l'Ingénu fut accordée le 3 septembre 1767 et le jour même le livre parut. Le succès fut immédiat : quatre mille exemplaires se vendirent en quelques jours, chiffre considérable pour l'époque. Mais le parti dévot, pris au dépourvu, réagit et le 17 septembre l'autorisation tacite fut retirée. Pendant ces péripéties, Voltaire joua l'habituelle comédie du désaveu. Il donna à choisir : ou bien l'Ingénu n'existait pas, ou bien, s'il existait, il n'était point de lui. Cependant, l'ouvrage n'avait pas besoin de cette publicité pour réussir. On connaît neuf éditions de 1767 et de maintes imitations, suites, parodies, adaptations théâtrales, ainsi que des traductions en allemand (1784), anglais (1786), russe (1789), qui accompagnaient les grands succès de librairie. Depuis lors, sa réputation fut presque égale à celle de Candide. (POMEAU, 1966 : 317) 
Voltaire présenta son récit comme histoire véritable, tirée des manuscrits du P. Quesnel. Vu les analogies, il s'inspira de l'Espion Américain en Europe, paru auparavant, mais il existe des preuves qu'il avait prémédité son ouvrage. Il nota d'abord un bref canevas pour résumer l'action et définit le statut des personnages secondaires et du protagoniste qu'il laissait mourir à la fin de l'histoire :

«Histoire de l'Ingénu, élevé chez les sauvages, puis chez les Anglais, instruit de la religion en Basse-Bretagne, tonsuré, confessé, se battant avec son confesseur. Son voyage à Versailles, chez frère Le Tellier son parent. Volontaire deux campagnes, sa force incroyable, son courage. Veut être capitaine de cavalerie, étonné du refus. Se marie, ne veut pas que le mariage soit un sacrement. Trouve très bon que sa femme soit infidèle parce qu'il l'a été. Meurt en défendant son pays. Un capitaine anglais l'assiste à la mort, avec un jésuite et un janséniste. Il les instruit en mourant. » (D'après POMEAU, 1966 : 318)

S'étant ainsi fixé un cadre d'ensemble, Voltaire évita que la variété des épisodes ne se perdît dans la dispersion. L'autographe du schéma initial, conservé dans le fonds de Pétersbourg, permet de mesurer l'étendue des modifications apportées. Suivant l'actualité contemporaine, l'affaire La Chalotais, Voltaire transporta l'action à l'époque de la Révocation de l'Édit de Nantes, les jésuites y jouèrent un rôle de premier plan.

Voltaire ne munit son texte d' aucune préface. Il s'adresse à un public assez large de liseurs de roman par la bouche du narrateur qui se porte explicitement garant de son récit. Dans les vingt chapitres, il présente deux milieux contrastés, la province et Paris, et met au centre du récit la formation intellectuelle d'un jeune homme par M. Gordon, un vieux janséniste enfermé à la Bastille. «Les âmes des deux captifs s'attachaient l'une à l'autre. Le vieillard savait beaucoup, et le jeune homme voulait beaucoup apprendre. » (VOLTAIRE, 1996 : 350) M. Gordon oriente son jeune ami vers la lecture et la conversation instructive, de plus en plus intéressante pour les deux intervenants. L'Ingénu se forme une bibliothèque composite, comprenant de petits livres de critique ou de brochures périodiques « où des hommes incapables de rien produire dénigrent les productions des autres » (VOLTAIRE, 1996 : 354), des livres sur l'histoire ancienne et celle de France, l'astronomie et d'autres sciences naturelles, des poésies, des traductions de tragédies grecques, quelques pièces du thêâtre français, etc. Son ami janséniste l'encourage à mettre par écrit ses réflexions qui parfois épouvantent son mentor :

« quoi! j'ai consumé cinquante ans à m'instruire, et je crains de ne pouvoir atteindre à bon sens naturel de cet enfant sauvage! je tremble d'avoir laborieusement fortifié des préjugés; il n'écoute que la simple nature. »(VOLTAIRE, $1996: 354)$

Dans cet intervalle, le Huron se transforme, il développe son jugement, il devient homme. Il cesse d'être une figure passive, victime livrée aux soins de sa famille d'accueil qui organisait son intégration dans la société au nom de la civilité et décidait de tout ("nommé l'Ingénu, converti, bâptisé, fiancé, envoyé à Versailles, sauvé par sa bien-aimée »). Le message de Voltaire vise encore à instruire les lecteurs : pour agrandir l'âme, il faut cultiver le bon sens naturel par la lecture critique des livres, bien choisis, de tous les domaines de la vie humaine et de 
l'histoire de l'humanité. Nous pouvons constater qu'il maintenait fermement ce qu'il avait dit ou écrit et que l'esprit scolaire, la fidélité aux classiques et à une culture littéraire encyclopédique en lui persistaient :

"Les vers qui parlaient d'amour portèrent à la fois dans l'âme de l'Ingénu le plaisir et la douleur. Ils lui parlaient tous de sa chère St. Yves. La fable des «Deux pigeons » lui perça le cœur ; il était bien loin de pouvoir revenir à son colombier. »(VOLTAIRE, 1996 : 355)

L'histoire s'achève par la mort de la belle St. Yves, dénouement pathétique qui représente le point faible du récit. Le vrai dénouement se trouve dans le ton plaisant voltairien qui récompense avec ironie tous les intérêts des personnes intéressées et le départ du jeune homme d'après les conseils qu' on lui donne :

«Mons. de Louvois vit enfin à bout de faire un excellent officier de l'Ingénu, qui a paru sous un autre nom à Paris et dans les armées, avec l'approbation de tous les honnêtes gens, et qui a été à la fois un guerrier et un philosophe intrépide. ». (VOLTAIRE, 1996 : 381)

Le jeune Huron civilisé peut continuer sa carrière en France sous un autre nom ! Comment peut-on devenir un bon Français sans être Français?

\section{Vivre libre en société civilisée}

Le Huron nommé l'Ingénu et la Péruvienne Zilia arrivèrent en France de l'Amérique, pays des Indiens, mais pas du même endroit. Il est évident que la civilisation péruvienne semblait plus exotique à un Français du temps des Lumières, que celle du Canada, pays en partie apparenté à la France. Mme de Graffigny décida donc de parfaire son livre en joignant l'utile - introduction historique et annotations explicatives en bas des pages, à l'agréable - missives sentimentales rédigées par la fille du Temple du Soleil à son fiancé éloigné. Le statut social des protagonistes ainsi que les causes de leur arrivée sur le territoire français diffèrent considérablement : Zilia, victime des conquistadors espagnols, se voit sauvée par Déterville, gentilhomme français ; le brave Huron y arrive pour découvrir le pays de ses aïeux, dont il parle couramment la langue. Zilia qui s'exprime d'abord en quipos apprend, au fur et à mesure, la langue française jusqu'à pouvoir traduire ses lettres en français, dans sa retraite. Bien que Déterville se fasse précepteur de Zilia, elle est plutôt autodidacte, elle s'instruit elle-même grâce à son don d'observation et de réflexion.

Les deux jeunes gens sont pleins de bonne foi et de bonne volonté. Curieux et courageux, ils veulent s'instruire, ils veulent comprendre comment vivent les Français. Les deux sont bien accueillis par les familles d'accueil qui représentent un échantillon de la société française, instruite depuis plus d'un siècle par la doctrine de la civilité qui doit respecter les règles et suivre les consignes. Cette société fermée et concentrée sur elle-même a du mal à accepter quelqu'un qui vient d'ailleurs. Pour un Français de l'Hexagone, un sauvage est inacceptable. Mais même les habitants des pays voisins du vieux continent, plus compréhensibles aux Français, sont incompréhensibles dans leurs coutumes, mœurs, religions, comportements... 
Ce qui est différent, c'est la réaction des protagonistes à des propositions d'assimilation dans cette société hostile. Zilia découvre au cours du roman la relativité des valeurs et cherche à s'intégrer dans la société française, tout en gardant son indépendance. L'Ingénu poursuit, sous un autre nom, sa carrière ailleurs. L'héroïne dépourvue de tout affronte son destin avec un pragmatisme ferme, le héros se laisse guidé... par qui ? par quoi ? L'arrivisme d'un Rastignac ? La différence des choix possibles correspond au choix générique fait par les créateurs de ces personnages romanesques, Mme de Grafigny préfère la subjectivité, le discours en lettres, Voltaire l'objectivité, le récit en chapitres. Zilia s'exprime par écrit, l'Ingénu en débattant avec M. Gordon. Mme de Graffigny fait goûter à ses lecteurs (et lectrices) du nouveau, Voltaire préfère suivre la tradition. Pourtant, c'est le plaisir de vivre qui subsiste :

«Le plaisir d'être, ce plaisir oublié, ignoré même de tant d'aveugles humains ; cette pensée si douce, ce bonheur si pur, je suis, je vis, j'existe, pourrait seul rendre heureux, si l'on s'en souvenait, si l'on en jouissait, si l'on en connaissait le prix. » (Lettre dernière, adressée par Zilia à Déterville). 


\section{BIBLIOGRAPHIE}

Beaumarchais Jean-Pierre de, Couty Daniel, Rey Alain (1994²), Dictionnaire des littératures de langue française, t. 2, Paris, Bordas, p. 1041-1042.

Biographie universelle ancienne et moderne, t. 18 (1817), Paris, L. G. Michaud, p. 262-264.

Biographie universelle ancienne et moderne, t. 47 (1827), Paris, L. G. Michaud, p. 466-512.

Coulet Henri (2014), Études sur le roman français au XVIII siècle, Paris, Champion.

Correspondance de Mme de Graffigny, 15 vol.(1985-2016), SHOWALTER English et al. (éds.), Oxford, Voltaire Foundation, Taylor Institution.

Dainard J. A., Allan Peter, ShOwalter English, Boursier Nicole, Curtis Judith, Kerslake L. C., Smith David W., Trott David A., Walker E. A. (1978), La correspondance de Madame de Graffigny, Dix-huitième Siècle, $\mathrm{n}^{\circ} 10$, Qu'est-ce que les Lumières ?, p. 379-394.

HERMAN Jan (2007), La scénographie des préfaces, in : GALLERON Ioana (éd.) L'Art de la préface au siècle des Lumières, Rennes, PUR.

Koulessa Rotraud von (1997), Françoise de Grafigny : Lettres d'une Péruvienne. Interpretation, Genese und Rezeption eines Briefromans aus dem 18. Jahrhundert, Stuttgart/Weimar, Metzle.

Koulessa Rotraud von (éd.), Lettres d'une Péruvienne (2014), Paris, Classiques Garnier.

LAFFONT Robert, BOMPIANI Valentino (1986), Dictionnaire biographique des Auteurs de tous les temps et de tous les pays, t. II, éd. Bouqins, Robert Laffont, Paris, p. 368-369

GRAFFIGNY Françoise de (2002), Lettres d'une Péruvienne, MALLINSON Jonathan (éd.), Oxford, Voltaire Foundation.

Voltaire (1996), L'Ingénu, in : Voltaire, Romans et contes, Paris, GarnierFlammarion, p. 323-381.

Pomeau René (1966), Note sur l'Ingénu, in : Voltaire, Romans et contes, Paris, Garnier-Flammarion, p. 317-322.

QUÉRARD Joseph-Marie (1829), La France littéraire ou Dictionnaire bibliographique des savants, historiens et gens de lettres de la France, ainsi que des littérateurs étrangers qui ont écrits en français, plus particulièrement pendant les XVII et XIX ${ }^{e}$ siècle, t. 3, Paris, Firmin Didot frères, p. 441-442.

QUÉRARD Joseph-Marie (1839), La France littéraire ou Dictionnaire bibliographique des savants, historiens et gens de lettres de la France, ainsi que des littérateurs étrangers qui ont écrit en français, plus particulièrement pendant les XVII et XIX ${ }^{e}$ siècle, t. 10, Paris, Firmin Didot frères, p. 276-457, éditions p. 323 ; imitations et parodies p. 392. 